\title{
Wolbachia segregation dynamics and levels of cytoplasmic incompatibility in Drosophila sechellia
}

\author{
S Charlat, P Bonnavion and H Merçot \\ Institut Jacques Monod, Laboratoire Dynamique du Génome et Evolution, CNRS-Universités Paris 6 \& 7, 2 Place Jussieu 75251 Paris \\ Cedex 05, France
}

\begin{abstract}
In Drosophila sechellia, the endocellular bacterium Wolbachia induces cytoplasmic incompatibility $(\mathrm{Cl})$ : in crosses involving infected males, a partial or complete embryonic mortality occurs unless the female bears the same Wolbachia. D. sechellia is known to harbour two Wolbachia variants, namely $w S h$ and $w S n$, closely related to $w \mathrm{Ha}$ and $w$ No, respectively, two strains infecting the populations of $D$. simulans from the Seychelles archipelago and New Caledonia. Strikingly, the two species show similar infection patterns: in $D$. sechellia, wSh can be present on its own or in double infection with $w S n$, but individuals carrying $w S n$ only do not occur; in $D$. simulans, wHa can be present on its own or in double infection with $w$ No, but individuals carrying $w$ No only do not occur, or occur at very low frequency. Previous
\end{abstract}

experiments on $D$. simulans showed that lines singly infected by $w$ No can be obtained by segregation, and stably maintained. Here we investigate this issue in $D$. sechellia through an 18 generation experiment, and show that wSn and $w$ Sh singly infected lines can arise by segregation. Using singly infected lines obtained in this experiment, we estimate the $\mathrm{Cl}$ intensities of $w S h$ and $w S n$ in $D$. sechellia, and compare these to the $\mathrm{Cl}$ intensities of the same Wolbachia injected into $D$. simulans. Our results do not suggest any consistent effect of the host species on the $\mathrm{Cl}$ induced by $w S h$. On the contrary, it seems that $w S n$ expression is repressed by host factors in $D$. sechellia.

Heredity (2003) 90, 157-161. doi:10.1038/sj.hdy.6800211

Keywords: Wolbachia; cytoplasmic incompatibility; symbiosis; endocellular bacteria; genetic conflicts; Drosophila

\section{Introduction}

In Drosophila sechellia, as in many other Arthropod species, the endocellular bacterium Wolbachia induces an intriguing form of male sterility, known as cytoplasmic incompatibility (CI) (reviewed in Hoffmann and Turelli, 1997; Charlat et al, 2002a). Males carrying the symbiont suffer a complete or partial loss of fertility when mated with uninfected females, but fertility is restored if the female harbours the same Wolbachia variant. Uninfected females thus suffer a fertility deficit relative to infected ones, the intensity of which depends on the frequency of infected males. The bacterium being transmitted by females only, through the egg cytoplasm, this highly deleterious effect on male hosts is not counter selected. On the contrary, infected females being fitter than uninfected ones, CI allows Wolbachia to invade uninfected host populations in a positive frequencydependent manner and then maintain itself (Caspari and Watson, 1959; Turelli and Hoffmann, 1995). In fact, CI is only one of the various means by which Wolbachia manipulates its host's reproduction for its own benefit (O'Neill et al, 1997; Stouthamer et al, 1999). The molecular basis of CI is currently unknown, but a model proposes that two bacterial functions at least are likely to be involved: $\bmod$ (for modification) would be a sort of

Received 15 November 2001; accepted 22 October 2002 poison, affecting paternal chromosomes before Wolbachia is shed from maturing sperm and resc (for rescue) would be a sort of antidote, saving the embryo from death when expressed in infected eggs (Werren, 1997; Poinsot et al, 2003)

$D$. sechellia is endemic from the Seychelles archipelago. It is known to carry two different Wolbachia strains: wSh and wSn (Rousset and Solignac, 1995; Bourtzis et al, 1996). These two variants are distinguishable by several molecular markers (Rousset and Solignac, 1995; Werren et al, 1995; Zhou et al, 1998; Charlat et al, 2002b). They belong to the two different main clades of Arthropods' Wolbachia (clades A and B), having diverged for about 60 million years. In laboratory strains, two different types of infected individuals can be found: those carrying wSh only (singly infected) and those carrying wSh and wSn (doubly infected). On the contrary individuals carrying wSn only are not observed (Rousset and Solignac, 1995).

$D$. simulans is very closely related to D. sechellia (Hey and Kliman, 1993; Kliman et al, 2001). It is known to harbour several Wolbachia strains, among which are $w \mathrm{Ha}$ and $w \mathrm{No}$, closely related to $w \mathrm{Sh}$ and $w \mathrm{Sn}$, respectively (Rousset and Solignac, 1995; Werren et al, 1995; Zhou et al, 1998; Charlat et al, 2002b). $w \mathrm{Ha}$ and $w$ No infect the $D$. simulans populations from New Caledonia and the Seychelles archipelago, where the infection pattern strikingly parallels the situation of $D$. sechellia: some individuals harbour only $w \mathrm{Ha}$, while others carry both $w \mathrm{Ha}$ and $w \mathrm{No}$, but individuals singly infected by $w \mathrm{No}$ are absent or very rare (James et al, 2002).

Experiments have shown that this pattern of infection in $D$. simulans is not because of the impossibility of $w \mathrm{No}$ 
lines being obtained by segregation from double infection, and subsequently maintained (Poinsot et al, 2000). Here we address the same issue in D. sechellia, through an 18-generation experiment, based on a similar procedure, and show that wSh as well as wSn singly infected lines can be obtained by segregation and stably maintained. Using $D$. sechellia singly infected lines as well as $D$. simulans lines artificially infected by wSh and wSn (Charlat et al, 2002b), we estimate CI levels in these two species. Our results do not suggest any consistent effect of the host species on the CI induced by wSh. By contrast, $w S n$ expression seems to be repressed by host factors in D. sechellia.

\section{Materials and methods}

\section{$D$. sechellia strains}

$S 9$ is a strain naturally infected by wSh (Bourtzis et al, 1996). Dsech is a strain infected by wSh+wSn, founded in the 1980s, originating from the Seychelles archipelago. DsechTC is an uninfected strain, obtained from Dsech by antibiotic treatment (Tetracycline).

Dse1 to Dse14 are 14 lines used in the segregation experiment, each initiated using one single female from the Dsech strain. Among the singly infected lines obtained by segregation from these doubly infected lines (see below), three have been used in subsequent CI assays, which took place 30 generations after the end of the segregation experiment.

\section{D. simulans strains}

STC is an inbred uninfected strain obtained by antibiotic treatment (Tetracycline) from the Seychelles strain, naturally infected by $w \mathrm{Ha}+w \mathrm{No}$, derived from flies collected on Mahe island (Seychelles archipelago) in 1981.

ASh and CSh are isofemale lines, infected by wSh, obtained by cytoplasmic injection from $D$. sechellia into the STC strain (Charlat et al, 2002b). ASn and BSn are isofemale lines, infected by $w S n$, obtained by cytoplasmic injection from $D$. sechellia into the STC strain (Charlat et al, 2002b). CI assays involving ASh, CSh, ASn and BSn took place 40 generations after the injection into $D$. simulans.

\section{Rearing conditions}

In order to ensure optimal conditions for the maintenance of Wolbachia infections, host strains were maintained at $25^{\circ} \mathrm{C}$, at low larval density, on axenic medium (David, 1962).

\section{Segregation experiment}

The present experiment followed a similar procedure to that of Poinsot et al (2000). At generation G0, one female from each of the 14 doubly infected lines (Dsech-1 to Dsech-14, infected by $w \mathrm{Sh}+w \mathrm{Sn}$ ) was crossed with two DsechTC males, before its infection status was checked by PCR. CI does not occur in such crosses, since males are not infected. Consequently, the hatching probabilities of the eggs laid by infected mothers do not depend on their infection status. From each of the 14 crosses, one G1 female was picked randomly and backcrossed with two DsechTC males, before its infection status was determined by PCR. A similar procedure was applied for 18 generations, allowing the total bacterial load as well as the proportion of wSh vs wSn to vary randomly.

\section{Wolbachia detection and identification}

The detection of Wolbachia and the distinction between the different Wolbachia variants were made by PCR. DNA was obtained according to O'Neill et al (1992) and the wsp gene was amplified according to Zhou et al (1998). Primer specificity allows distinguishing wSh (primer $178 \mathrm{~F}$ and 691R) from $w S n$ (primers $183 \mathrm{~F}$ and 691R).

\section{Measurement of embryonic mortality}

Embryonic mortality was measured in D. simulans and D. sechellia using individual crossings between males aged 3-4 days and females aged 4-7 days. Mating was observed, and crosses where copulation lasted for less than 15 min were discarded. Inseminated females were individually placed at $25^{\circ} \mathrm{C}$, on axenic medium, coloured with neutral red. $D$. simulans females were left to lay for $48 \mathrm{~h}$ and $D$. sechellia females for $96 \mathrm{~h}$, owing to their lower fecundity. Laying boxes were left for an additional $24 \mathrm{~h}$ at $25^{\circ} \mathrm{C}$ so that all viable embryos could hatch, and finally placed at $4{ }^{\circ} \mathrm{C}$ until egg counting. Embryonic mortality was then determined as the percentage of unhatched eggs. Laying boxes with less than 20 eggs were discarded. In $D$. sechellia, average egg number was 46.9, ranging from 20 to 96 . In $D$. simulans, average egg number was 86, ranging from 31 to 142 . For crosses showing $0 \%$ hatching, a fertility test was performed by crossing each parent with individuals harbouring a compatible infection. This procedure allows to distinguish between crosses where CI is $100 \%$ and crosses involving intrinsically sterile individuals. Crosses with one sterile parent were discarded. Finally, the infection status of parents was checked by PCR.

\section{$\mathrm{Cl}$ intensity}

$\mathrm{CI}$ intensity (or CI level) is defined here as the percentage of embryos that do not hatch because of CI, in crosses between infected males and uninfected females. CI intensity experiments thus involve crosses between infected males and uninfected females (strain DsechTC in D. sechellia, strain STC in D. simulans). In each experiment, control crosses, involving uninfected males, were also performed, in order to determine the control cross mortality (CCM). This allows the calculation of a corrected $\mathrm{CI}\left(\mathrm{CI}_{\text {corr }}\right)$, taking into account the embryonic mortality not caused by CI. If EM stands for the observed embryonic mortality, $\mathrm{CI}_{\mathrm{corr}}=(\mathrm{EM}-\mathrm{CCM}) /(1-\mathrm{CCM})$ (Poinsot et al, 1998).

\section{Statistical analysis}

All statistical tests were performed on SAS (1989, version 6.12) after root arcsine transformation.

\section{Results}

\section{Segregation rates}

The rate of segregation from doubly to singly infected cytoplasm was monitored through 18 generations, in conditions allowing the proportion of wSh and wSn, as well as the total bacterial load, to vary stochastically. The loss of wSh occurred in four lines out of 14 (Dse5 at generation 4, Dse6 and Dse8 at generation 14, Dse2 at generation 18). The loss of wSn occurred in only one line (Dse14, at generation 2). The complete loss of infection did not occur. At the end of the experiment, the respective proportions of doubly infected, wSh and 
$w S n$ lines were $64.3,7.1$ and $28.6 \%$. In total, 230 crosses involved females still bearing wSh. Among these, wSh was lost in four crosses, making an overall loss rate of $1.7 \%$. Similarly, wSn was lost in one cross out of 236 involving females still bearing this variant, making an overall loss rate of $0.42 \%$. These two rates are not significantly different (Fisher's exact test: $P=0.211)$

\section{$\mathrm{Cl}$ intensity in $D$. sechellia and $D$. simulans}

The CI intensity of $w$ Sh was estimated in two D. sechellia lines: S9 (naturally singly infected) and Dse14-Sh (obtained by segregation) and in two $D$. simulans lines: ASh and CSh (obtained by cytoplasmic injection (Charlat et al, 2002b)). The results are summarized in Table 1a. As expected, $w$ Sh was found to induce CI in all lines in both species: $t$-test comparisons with control crosses (Table 1c) are all significant, with $P<0.001$ (not shown). The host effect was investigated by ANOVA (Table 2a). The Line factor was found significant $(P=0.0028)$. Indeed, important between-line variations occur in $D$. simulans $\left(\mathrm{CI}_{\text {corr }}=78.7\right.$ and $50.0 \%$ for ASh and CSh, respectively), which are consistent with previous observations (Charlat et al, 2002b). Owing to this Line effect, the two species are not found to be significantly different, although the means are more than $20 \%$ apart $\left(\mathrm{CI}_{\text {corr }}=90 \%\right.$ in $D$. sechellia vs $67 \%$ in $D$. simulans). In other words, the between-species variation is not significantly higher than the between-line variation. Multiple comparisons, realised through the LSMEAN/TDIFF statement in SAS (1989), lead to similar conclusions: $S 9$ and $A S h$, although belonging to $D$. sechellia and $D$. simulans, respectively, do not differ significantly (not shown).

A similar experiment was carried out with $w \mathrm{Sn}$. CI intensity was estimated in two D. sechellia lines: Dse5-Sn and Dse6-Sn (obtained by segregation) and in two $D$. simulans lines: $A S n$ and $B S n$ (obtained by cytoplasmic injection (Charlat et al, 2002b)). The results are summarized in Table 1b. As expected, wSn was found to induce $\mathrm{CI}$ in all lines in both species: $t$-test comparisons with control crosses are all significant, with $P<0.001$ (not
Table 2 CI intensity, ANOVAs

\begin{tabular}{lrccll}
\hline Source & $d f$ & Mean square & $F$ & F denominator & Pr $>F$ \\
\hline (a) $w$ Sh & & & & & \\
Species & 1 & 2.1308 & 4.10 & Line & NS \\
Line & 2 & 0.5211 & 6.40 & Error & 0.0028 \\
Error & 71 & 0.0814 & - & - & - \\
& & & & & \\
(b) $w$ Sn & & & & & \\
Species & 1 & 1.6577 & 25.15 & Error & 0.0001 \\
Line & 2 & 0.0524 & 0.80 & Error & NS \\
Error & 71 & 0.0659 & - & - & - \\
\hline
\end{tabular}

Line is nested within Species.

shown). As shown in Table $2 b$, there does not seem to be any intraspecific variation in this case (Line factor nonsignificant). By contrast, the Species factor is found to be significant $(P=0.0001)$. Thus, $w \mathrm{Sn}$ in $D$. sechellia induces a lower $C I$ than in $D$. simulans (mean $\mathrm{CI}_{\mathrm{corr}}=39 \%$ vs 64\%). Multiple comparisons lead to similar conclusions: within each species, the lines do not differ, but all interspecific comparisons are significant (not shown).

\section{Discussion}

\section{Segregation rates}

The rates of loss of $w S h$ and $w$ Sn from initially doubly infected cytoplasm were followed through 18 generations, in conditions where the effect of CI (the selection of doubly infected embryos by doubly infected males) was relaxed. At every generation, infected females were backcrossed to uninfected males harbouring the same genomic background. The two types of singly infected cytoplasm were obtained and stably maintained. The transmission efficiencies of the two variants were not found to differ significantly. This experiment demonstrates that the absence of lines singly infected by $w S n$ in $D$. sechellia is not because of a perfect transmission of this

Table $1 \mathrm{CI}$ intensity in D. sechellia and D. simulans: descriptive statistics

\begin{tabular}{|c|c|c|c|c|c|c|c|}
\hline Host species & Male & Female & $\mathrm{n}$ crosses & n eggs & $E M(\%)$ & $C I_{\text {corr }}(\%)$ & SE $(\%)$ \\
\hline \multicolumn{8}{|l|}{ (a) $w \mathrm{Sh}$} \\
\hline $\begin{array}{l}\text { D. sechellia } \\
\text { D. sechellia } \\
\text { D. simulans } \\
\text { D. simulans }\end{array}$ & $\begin{array}{l}\text { Dse14-Sh (wSh) } \\
\text { S9 (wSh) } \\
\text { ASh (wSh) } \\
\text { CSh (wSh) }\end{array}$ & $\begin{array}{l}\text { DsechTC } \\
\text { DsechTC } \\
\text { STC } \\
\text { STC }\end{array}$ & $\begin{array}{l}19 \\
17 \\
23 \\
16\end{array}$ & $\begin{array}{r}857 \\
713 \\
1978 \\
1230\end{array}$ & $\begin{array}{l}92.8 \\
90.4 \\
82.9 \\
59.5\end{array}$ & $\begin{array}{l}90.4 \\
87.3 \\
78.7 \\
50.0\end{array}$ & $\begin{array}{l}2.58 \\
3.89 \\
3.82 \\
7.67\end{array}$ \\
\hline \multicolumn{8}{|l|}{ (b) $w \mathrm{Sn}$} \\
\hline $\begin{array}{l}\text { D. sechellia } \\
\text { D. sechellia } \\
\text { D. simulans } \\
\text { D. simulans }\end{array}$ & $\begin{array}{l}\text { Dse5-Sn (wSn) } \\
\text { Dse6-Sn (wSn) } \\
\text { ASn }(w S n) \\
B S n(w S n)\end{array}$ & $\begin{array}{l}\text { DsechTC } \\
\text { DsechTC } \\
\text { STC } \\
\text { STC }\end{array}$ & $\begin{array}{l}16 \\
23 \\
19 \\
17\end{array}$ & $\begin{array}{r}908 \\
1255 \\
1831 \\
1667\end{array}$ & $\begin{array}{l}49.8 \\
55.7 \\
70.4 \\
72.4\end{array}$ & $\begin{array}{l}34.1 \\
41.7 \\
63.0 \\
66.0\end{array}$ & $\begin{array}{l}6.72 \\
5.33 \\
3.77 \\
3.85\end{array}$ \\
\hline \multicolumn{8}{|c|}{ (c) Control crosses } \\
\hline $\begin{array}{l}\text { D. sechellia } \\
\text { D. simulans }\end{array}$ & $\begin{array}{l}\text { DsechTC (uninfected) } \\
\text { STC (uninfected) }\end{array}$ & $\begin{array}{l}\text { DsechTC } \\
\text { STC }\end{array}$ & $\begin{array}{l}28 \\
33\end{array}$ & $\begin{array}{l}1097 \\
2589\end{array}$ & $\begin{array}{l}24.9 \\
19.5\end{array}$ & - & $\begin{array}{l}4.11 \\
2.37\end{array}$ \\
\hline
\end{tabular}

EM: raw embryonic mortality, $\mathrm{CI}_{\text {corr }}$ : corrected CI, SE: Standard Error. 
variant, or to its lethality when present on its own. Interestingly, the complete loss of Wolbachia infection did not occur, suggesting that the total bacterial load did not decrease during the experiment. In other words, it seems that the emergence of singly infected cytoplasm was rather due here to stochastic variations in the proportions of the two variants than to a decrease of bacterial population size.

In a similar experiment realised in $D$. simulans, Poinsot et al (2000) followed the segregation dynamics of $w \mathrm{Ha}$ and wNo through 18 generations. Although the experiments were not performed together, a comparison can be made with the present results. The distribution of the various infection types at the end of the experiment with $D$. simulans can be considered: $33.3 \%$ doubly infected, $24.3 \%$ singly infected by $w \mathrm{Ha}, 12.1 \%$ singly infected by $w$ No and $30.3 \%$ uninfected $(n=33)$. These proportions contrast with those observed in $D$. sechellia (Fisher's exact test: $P=0.014$ ). In $D$. simulans, not only did singly infected lines of both types occur more frequently, but uninfected ones did as well. These results suggest that $w S h$ and $w S n$ in $D$. sechellia are more efficiently transmitted, than are $w \mathrm{Ha}$ and $w \mathrm{No}$ in D. simulans.

\section{$\mathrm{Cl}$ intensity in $D$. sechellia}

The CI intensity of wSh and wSn was estimated in their natural host $D$. sechellia. Such estimations have been made previously for wSh, naturally present as a single infection (Rousset and Solignac, 1995; Bourtzis et al, 1996). In these two studies, the raw embryonic mortality in crosses between wSh males and uninfected females was, respectively, 71 and $70 \%$. If one takes into account the control crossmortality (Poinsot et al, 1998), the corrected CI levels are 62 and $66 \%$, which is lower than what we observed $(90.4 \%$ in Dse14-Sh and $87.3 \%$ in 59 , the line used by Bourtzis et al, 1996). These differences are likely to be because of environmental factors, such as larval density, which are known to affect CI levels (Sinkins et al, 1995). Interestingly, we found that wSh expresses the same CI intensity in Dse14-Sh and S9, although the first line derives from the segregation experiment and the second is naturally singly infected. This is consistent with the view that natural singly infected lines derive from doubly infected ones through imperfect transmission.

Before our experiment, D. sechellia lines singly infected by $w S$ nere not available. However, the CI intensity of wSn had been indirectly investigated by crossing doubly infected males with females bearing only wSh (Rousset and Solignac, 1995). In such crosses, the raw embryonic mortality was $35 \%$. In this case, $\mathrm{CI}_{\text {corr }}$ is also $35 \%$ since the control crossmortality is $0 \%$. This value is very close to the ones we observed in crosses involving males singly infected by wSn $(34.1 \%$ in Dse5-Sn and $41.7 \%$ in Dse6-Sn), suggesting that the two variants in double infections do not affect each other (the intensity of one variant does not depend on the presence or absence of the other), consistent with several previous observations (Merçot et al, 1995; Rousset and Solignac, 1995; Rousset et al, 1999).

Based on our estimations of transmission efficiency and CI intensity, one can calculate the equilibrium frequencies expected under Hoffmann and Turelli's model (1997). Assuming (i) that neither wSh nor wSn affect host fitness (apart from CI) and (ii) that the CI effects of the two strains are additive, the expected frequencies are the following: $99.17 \%$ doubly infected, $0.63 \%$ wSh, $0.19 \%$ wSn and $0.001 \%$ uninfected. Thus, $w S h$ flies are expected to be three times more frequent than $w S n$, but both types of singly infected cytoplasms are expected to be rare. The very sparse data available on the frequencies of singly infected lines in the wild suggest that they might be more frequent (Rousset and Solignac, 1995). Two different factors might explain this discrepancy: our experimental conditions allow the expression of high CI levels and high transmission efficiency, both factors that increase the expected equilibrium frequencies of the double infection, and reduce the expected frequency of single infections. Gathering parameter estimates from the wild, together with additional data on the frequencies of the various infection types, might clarify this issue.

\section{Evidence for a host effect}

In order to investigate the possibility of host control on wSh and/or wSn, CI assays were realised in the foreign host $D$. simulans, using transinfected lines obtained by cytoplasmic injection (Charlat et al, 2002b).

Concerning wSh, the results do not suggest any variations between the two hosts. Within $D$. simulans, a significant difference was detected between lines. Previous results indicate that such differences do not remain stable over time, suggesting that they might be caused by uncontrolled environmental factors (Charlat et al, 2002b). Whatever the causal factor, this intraspecific variation in $D$. simulans lowers the statistical power of our comparison. Indeed, although the mean $\mathrm{CI}_{\text {corr }}$ in the two species is more than $20 \%$ apart, the difference is not significant. This highlights the importance of using more than one line to investigate such effects, in order to avoid erroneous conclusions.

On the contrary, wSn was shown to induce different CI levels in the two species. Consistent with previous observations, there does not seem to be any betweenline variation for $w S$ nithin $D$. simulans (Charlat et al, $2002 b)$. Thus, $w$ Sn expresses a higher CI in D. simulans than in its natural host (mean $\mathrm{CI}_{\text {corr }}=39 \%$ in $D$. sechellia vs $64 \%$ in D. simulans). Overall, it appears that the differences between $D$. simulans and $D$. sechellia regarding the CI intensity of $w \mathrm{Sn}$ and $w$ No are mainly because of host effects, and not because of divergence between the two strains. Indeed, while the present results show that wSn induces a lower CI in D. sechellia than in $D$. simulans, previous experiments suggest that wSn and $w$ No, within $D$. simulans, induce similar levels of mortality (Charlat et al, 2002b). Under this view, one could predict that $w$ No and $w S n$ should induce the same CI levels in $D$. sechellia. This remains to be investigated.

It is notable that the CI assays realised on $w S h$ and $w S n$ did not lead to identical conclusions regarding the host controls on CI levels: it appears that $w \mathrm{Sn}$ only is repressed in $D$. sechellia. Indeed, if there might be a slight host effect for wSh, undetected in the present experiment, the tendency is rather that of a higher level of expression in $D$. sechellia than in D. simulans, suggesting that the host control observed here is Wolbachia strain-specific. 


\section{Acknowledgements}

We thank Kostas Bourtzis for providing the $S 9$ strain and to the 'Population Génétique et Evolution' group for providing the Dsech strain. We also thank Francis Jiggins for commenting on this manuscript, Mélanie Baril for a helpful contribution to the experiment and Valérie Delmarre and Chantal Labellie for technical assistance.

\section{References}

Bourtzis K, Nirgianaki A, Markakis G, Savakis C (1996). Wolbachia infection and cytoplasmic incompatibility in Drosophila species. Genetics 144: 1063-1073.

Caspari E, Watson GS (1959). On the evolutionary importance of cytoplasmic sterility in mosquitoes. Evolution 13: 568-570.

Charlat S, Bourtzis K, Merçot H (2002a). Wolbachia-induced cytoplasmic incompatibility. In: Seckbach J (ed) Symbiosis. Kluwer Academic Publisher: Dordrecht, The Netherlands, pp 623-644.

Charlat S, Nirgianaki A, Bourtzis K, Merçot H (2002b). Evolution of Wolbachia-induced cytoplasmic incompatibility in Drosophila simulans and D. sechellia. Evolution 56: 17351742.

David J (1962). A new medium for rearing Drosophila in axenic conditions. Dros Inf Ser 93: 28.

Hey J, Kliman RM (1993). Population genetics and phylogenetics of DNA sequence variation at multiple loci within the Drosophila melanogaster species complex. Mol Biol Evol 10: 804-822.

Hoffmann AA, Turelli M (1997). Cytoplasmic incompatibility in insects. In: O'Neill SL, Hoffmann AA, Werren JH (eds). Influential Passengers: Inherited Microorganisms and Arthropod Reproduction. Oxford University Press: Oxford, pp 42-80.

James AC, Dean MD, McMahon ME, Ballard JWO (2002). Dynamics of double and single Wolbachia infections in Drosophila simulans from New Caledonia. Heredity 88: 182189.

Kliman RM, Andolfatto P, Coyne JA, Depaulis F, Kreitman M, Berry AJ, Mccarter J, Wakeley J, Hey J (2001). The population genetics of the origin and divergence of the Drosophila simulans complex species. Genetics 156: 1913-1931.

Merçot H, Llorente B, Jacques M, Atlan A, Montchamp-Moreau C (1995). Variability within the Seychelles cytoplasmic incompatibility system in Drosophila simulans. Genetics 141: 1015-1023.

O'Neill SL, Giordanno R, Colbert AME, Karr TL (1992). 16S rRNA phylogenetic analysis of the endosymbionts associated with cytoplasmic incompatibility in insects. Proc Natl Acad Sci USA 89: 2699-2702.

O'Neill SL, Hoffmann AA, Werren JH (1997). Influential Passengers: Inherited Microorganisms and Arthropod Reproduction. Oxford University Press: Oxford.

Poinsot D, Bourtzis K, Markakis G, Savakis C, Merçot H (1998). Wolbachia transfer from Drosophila melanogaster into $D$. simulans: host effect and cytoplasmic incompatibility relationships. Genetics 150: 227-237.

Poinsot D, Charlat S, Merçot H (2003). On the mechanism of Wolbachia-induced cytoplasmic incompatibility: confronting the models to the facts. BioEssays 25: in press.

Poinsot D, Montchamp-Moreau C, Merçot H (2000). Wolbachia segregation rate in Drosophila simulans naturally bi-infected cytoplasmic lineages. Heredity 85: 191-198.

Rousset F, Braig HR, O’Neill SL (1999). A stable triple Wolbachia infection in Drosophila with nearly additive incompatibility effects. Heredity 82: 620-627.

Rousset F, Solignac M (1995). Evolution of single and double Wolbachia symbioses during speciation in the Drosophila simulans complex. Proc Natl Acad Sci USA 92: 6389-6393.

SAS (1989). STAT User's Guide, version 6, 4th edn. SAS Institute: Cary, NC.

Sinkins SP, Braig HR, O'Neill SL (1995). Wolbachia superinfections and the expression of cytoplasmic incompatibility. Proc $R$ Soc London Ser B 261: 325-330.

Stouthamer R, Breeuwer JAJ, Hurst GDD (1999). Wolbachia pipientis: microbial manipulator of Arthropod reproduction. Annu Rev Microbiol 53: 71-102.

Turelli M, Hoffmann AA (1995). Cytoplasmic incompatibility in Drosophila simulans: dynamics and parameter estimates from natural populations. Genetics 140: 1319-1338.

Werren JH (1997). Biology of Wolbachia. Annu Rev Entomol 42: 587-609.

Werren JH, Zhang W, Guo LR (1995). Evolution and phylogeny of Wolbachia, reproductive parasites of arthropods. Proc $R$ Soc London Ser B 251: 55-63.

Zhou W, Rousset F, O'Neill SL (1998). Phylogeny and PCRbased classification of Wolbachia strains using wsp gene sequences. Proc $R$ Soc London Ser B 265: 509-515. 